\title{
FPGA Realization for Baseline Wander Noise Cancellation of ECG Signals using Wavelet Transform
}

\author{
Ashraf Mohammed Ali Hassan \\ Department of Electronics and Communication Engineering, Faculty of Engineering, October University for \\ Modern Sciences and Arts
}

\begin{abstract}
Baseline Wander (BW) is a common noise in electrocardiogram (ECG). To effectively correct and to preserve more underlying components of an ECG signal, a powerful tool for removal of BW noise from various signals was introduced. This paper presented the discrete wavelet to get rid of that noise. This method is based on comparing signal with discrete multi-rate filter banks. A multi-level decomposition was performed on the noisy signal and then the splitting into low sub-bands and a high pass band sub-bands called detail level was performed. After that, the analysis of the details level and the identifying of a suitable threshold technique were done. Reconstruction of the signal was done through the calculation of the detail coefficients. Finally, the difference between the original signal and the reconstructed signal was calculated. The proposed technique was compared with the previous techniques in this domain of search. The algorithm was tested using Matlab tool. The results showed that the proposed filter could more effectively extract baseline wander from ECG signal and affect the morphological feature of ECG signal considerably less than both the traditional moving average filter and adaptive filter did. The results showed also that this proposed technique achieved excellent results in terms of Mean Square Error (MSE) and convergence rate rather than the previous approaches. This paper also introduced the efficient realization of the proposed approach using FPGA. The proposed method was verified by FPGA (Xilinx Virtex-7 XC7VX690T) realization, revealing its effectiveness in real-time applications.
\end{abstract}

\section{General Terms}

Sub-band- Threshold technique - Matlab - FPGA.

\section{Keywords}

Baseline Wander - Electrocardiogram -Filter -Convergence Rate.

\section{INTRODUCTION}

Electrocardiogram (ECG) signals are usually contaminated by baseline wander (BW), which is commonly caused by electrode-skin impedance changes due to perspiration, patient movement [1]. In addition, BW severely influences computerbased processing. Therefore, it is necessary to reduce noise such as BW before the ECG signal is automatically analyzed by computer and finally interpreted by a cardiologist [2]. Figure 1 shows the typical ECG signal. In this paper the main target to use this technique to enhance the reduction of the noise of ECG signal which is considered an important method can help in the biomedical engineering because heart disease is the leading of mortality in the world. The electrocardiogram is widely used for the diagnoses of these diseases because it's a noninvasive way to establish clinical diagnosis of heart disease [3]. The modern society is today threatened by an incipient health care delivery crisis caused by the current demographic and lifestyle trends [4]. The ECG provides information about the heart. This signal is used in the extraction of vital characteristics [5]. In studying such type of signals, wavelet transform is very useful. The most striking waveform when considering the ECG is QRS wave complex, which gives the $\mathrm{R}$ wave peak which is time-varying [6]. Wavelet transform (WT) is a powerful time-frequency signal analysis tool and it is used in a wide variety of applications [7]. The question is how to use the Wavelet Transform for the purpose of getting rid of Baseline Wander ECG signal? The important issue is to get a reconstructed signal with the least error can be accomplished. A moving average filter [8], [9] and wavelet translation [10] were often used to construct a high-pass filter. However, a high-pass filter would unavoidably introduce distortions in various parts of the ECG signal, especially in the ST segment due to the spectra of the ST segment that overlaps the spectra of BW [11], [12]. The developed algorithm initially decomposes the original ECG signal by using Discrete Wavelet Transform [13]. The ECG signals for the present study are collected from the T-wave alternates challenge database on Physionet.org, twa18 and twa34 are chosen for experiments. Because ECG signal of the twa34 channel I has an almost (perceptually) isoelectric level baseline, a standard ECG signal is replaced by a segment of an ECG signal from the twa34 channel I. The chosen standard ECG signal lasts $6 \mathrm{~s}$ and holds 11 ECG cycles. The ECG signal of the twa18 channel III is severely contaminated by BW. Thus the ECG signal of the channel III is used as a real contaminated ECG signal to verify the performance of the proposed method [2]. The paper is organized as follow: Section 2 explains the related work, section 3 shows the proposed technique with analysis, section 4 demonstrates the simulation results, section 5 shows the FPGA realization and section 6 introduces the conclusion.

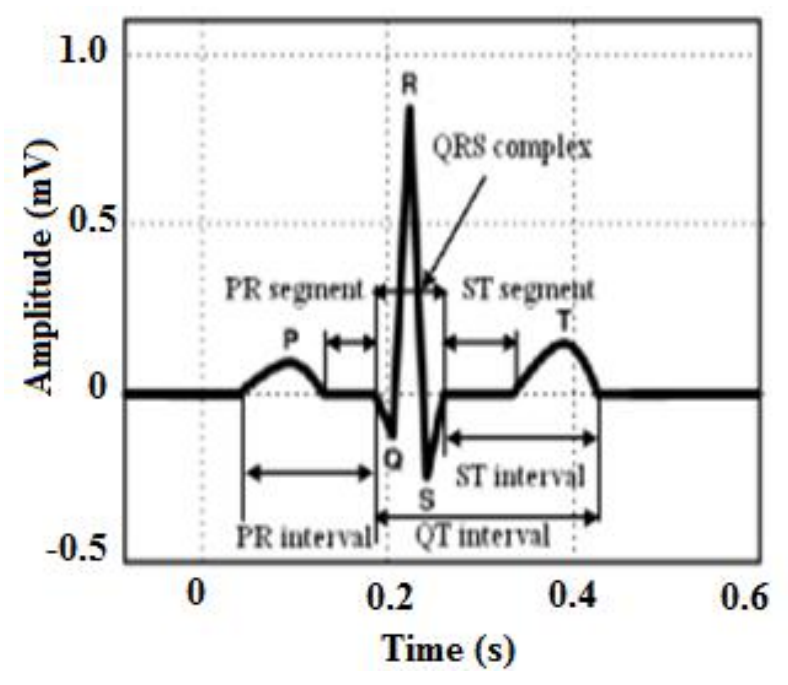

Fig. 1 Typical ECG signal 


\section{RELATED WORK}

One of the previous methods is a simple and novel filtering method based on statistical and weighted moving average filter. Supposed $\mathrm{a}$ and $\mathrm{b}$ are the minimum and maximum of all sample values within a moving window, respectively. First the whole region $[\mathrm{a}, \mathrm{b}]$ is divided into $\mathrm{M}$ equal sub-regions without overlap. Second, three sub-regions with the largest sample distribution probabilities are chosen (except $\mathrm{M}<3$ ) and incorporated into one region, denoted as $\left[\mathrm{a}_{0}, \mathrm{~b}_{0}\right]$ for simplicity. Third for every sample point in the moving window its weight is set to 1 if its value falls in $\left[a_{0}, b_{0}\right]$; otherwise, its weight is 0 . Last all sample points with weight 1 are averaged to estimate the baseline [2]. Another method used in filtering BW is using an adaptive filter. The adaptive noise cancellation configuration is as shown in Figure (2). The adaptive filter coefficients adapt to cause the error signal to be a noiseless version of the signal s(n) [14], [15], [16].

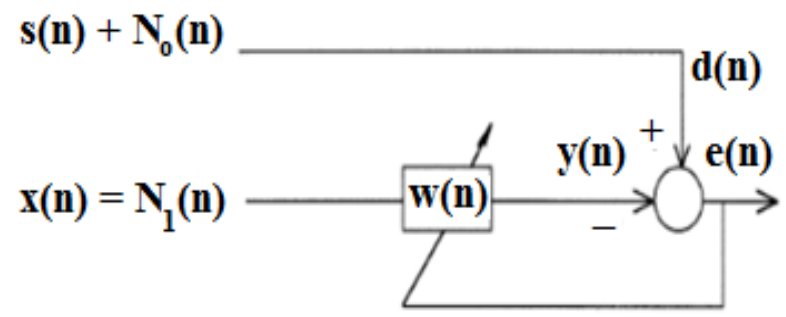

Fig.2 Noise cancellation using adaptive filter

Figure 2 shows an adaptive filter that has a primary input which is an ECG signal $s(n)$ with additive noise N1(n). Meanwhile the reference input is noise NO(n), possibly recorded from another generator of noise N1(n) [17], [18], [19].

\section{THE PROPOSED TECHNIQUE}

The technique is the Discrete Wavelet Transform (DWT). First the approximation and detail coefficients are obtained by performing multi-level wavelet decomposition. Figure 3 shows the proposed design

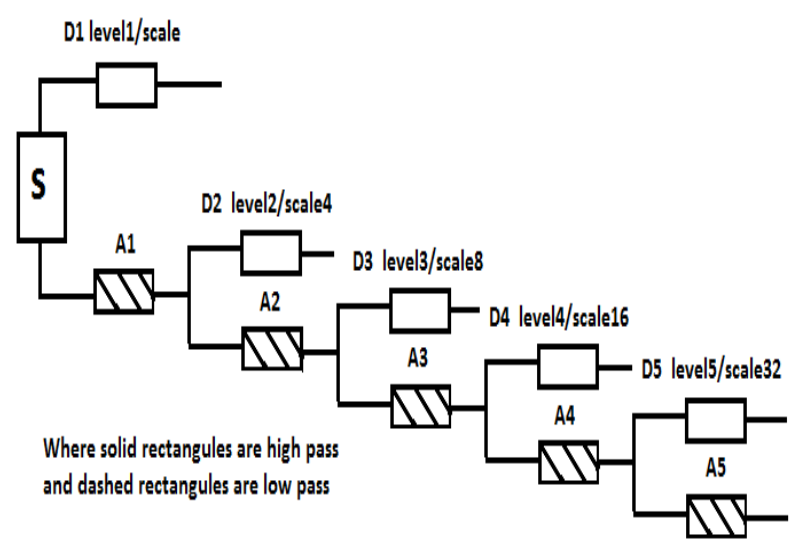

Fig. 3 The proposed design

Here in this design, a signal $\mathrm{S}$ produces low-pass and high pass sub-bands by using low and high pass filters. Depending on Nyquest criteria, half of the samples are discarded after filtering. The filters have a small number of coefficients and results in good computational performance. These filters also have the ability to reconstruct the sub-bands while canceling any aliasing that occurs due to down sampling for the next level of decomposition signal. The low-pass sub-band A1 is irrelatively filtered by the same technique to yield narrow subbands. DWT splits the signal into a low-pass sub-band which is called the approximation level and a high sub-band which is called detail level. Second, we analyze the detail and identify a suitable threshold technique. There are four main techniques available in MATLAB which they are universal, SureShrink or the "rigrsure" method, Hersure threshold and minimizing threshold. The universal is the simplest one to compute while the other three are manually computed. There're soft and hard threshold Both agree in that; the coefficients with the magnitudes less than the threshold magnitude are set to zero. In the hard threshold, the coefficients larger than the threshold value are left unchanged. Third, threshold the detail coefficients and reconstruct the signal. The entire process of threshold and reconstructing the signal from the new coefficients can be done using a single function as shown in the following section. The last step is to subtract the reconstructed signal from the original signal so we can evaluate the error in the technique the paper introduces.

\subsection{Amplitude modulation baseline wander}

Both the patient's movement and the perspiration between electrode and skin unavoidably cause an electrode-skin impedance change. As a result, similar to the amplitude modulation in communication, the amplitude of ECG signal, which is acquired through the electrode, varies with the changing impedance. This kind of BW is simulated from the Gaussian model [18].

$b_{1}(i)=A \cdot \exp \left(-s(i-p)^{\wedge} 2\right)$

Where $\mathrm{b}_{\mathrm{l}}(i)$ is the $i^{\text {th }} \mathrm{B}_{\mathrm{L}}$ value, $A$ is the $\mathrm{B}_{\mathrm{L}}$ peak with an appropriate peak time $p$, and $s$ is the amplitude change rate of $\mathrm{B}_{\mathrm{L}}$. In this experiment, $A, p$, and $s$ were set as $3.5 \mathrm{mV}, 2 \mathrm{~s}$, and 2.0 , respectively. Obviously the patient's respiration may lead to the periodic change of the BL curve simulated this using a sinusoidal model [18]:

$$
b_{1}(i)=A \cdot \sin (2 \pi f i)
$$

The $b_{1}$ simulated by Eq. (2) is a determinate signal. In fact, due to much random interference, $b_{1}$ should not be a sinusoid. To gain this 'real' BW-like sinusoid, two methods are used. One method involves normal distribution noise [20], [21]. These two kinds of noise represent the real BW presented in ECG signals much better than Eq. (2) that models the BW.

\subsection{Traditional high-pass filter}

The main types to describe linear or nonlinear high-pass filters are infinite-impulse response (IIR) and finite impulse response (FIR) [22]. The performance of IIR filters is generally unacceptable. This is because a nonlinear phase response introduces distortion into an ECG signal. Sampling rate decimation could reduce FIR complexity. Both the moving average filter and wavelet package translation are discussed here.

A moving average filter can be described as

$$
y(n)=\frac{1}{2 N+1} \sum_{i=-N}^{N} x(n+i)
$$

Where $x(n)$ and $y(n)$ are input signal and output signal of the moving average respectively, and $N$ is the observation 
window length and is equal to $2 N+1$. BL can be estimated using Eq. (3). Based on Eq. (3), a high-pass filter is formed:

$$
z(n)=x(n)-y(n)
$$

Where $z(n)$ is the output signal of the high-pass filter. The high-pass filter based on moving average is called MA in this paper. From Fig. 1, it is obvious that the BL values estimated from the P-R segment (between $0.3 \mathrm{~s}$ and $0.6 \mathrm{~s}$ ) are very close to real baseline values, while the BL values estimated from segments including QRS complex and $\mathrm{T}$ wave are far away from the real baseline. Therefore, if an observation window covers some sample points with extreme amplitudes, an ECG signal would be distorted after the ECG signal is filtered using Eqs. (3) and (4).

\subsection{Wavelet packet transform}

Wavelet package translation (WPT) was presented by Coifman and Wickerhauser as a generalized family of multiresolution orthogonal or biorthogonal bases [15]. As a result, WPT can obtain some finer frequency bands than wavelet transformation. Therefore, WPT has been widely used in biomedical signal analysis [16]. Given a finite energy signal with scaling space $\mathrm{U}_{0}{ }^{0}$ WPT might decompose $\mathrm{U}_{0}{ }^{0}$ into small subspaces $\mathrm{U}_{\mathrm{j}}^{\mathrm{n}}$ in a dichotomous way. $\mathrm{U}_{\mathrm{j}}^{\mathrm{n}-1}$ shows the $n^{\text {th }}$ subspace at the $j^{\text {th }}$ resolution level. The dichotomous way is realized using the following recursive scheme:

$U_{j+1}^{n}=U_{j}^{2 n} \oplus U_{j}^{2 N+1}, N=0,1,2, . .2^{-j}-1$

Where $j(j=-1,-2,-3, \ldots)$ is the resolution level and ' $\oplus$ ' denotes orthogonal decomposition. $U_{j+1}^{n}, U_{j}^{2 n}, U_{j}^{2 N+1}$ which are three close spaces corresponding to $u_{n}(t), u_{2 n}(t)$ and $u_{2 n+1}$ (t) respectively. $U_{n}(t)$ satisfies

$$
\left\{\begin{array}{c}
u_{2 n}(t)=\sqrt{2} \sum_{k=Z} h(k) u_{n}(2 t-k) \\
u_{2 n+1}(t)=\sqrt{2} \sum_{k=Z} g(k) u_{n}(2 t-k)
\end{array}\right\}
$$

Where $u_{0}(t)$ is identified with the scaling function $\varphi$ and $u_{1}(t)$ with the mother wavelet $\psi . h(k)$ and $g(k)$ are the coefficients of the low-pass and high-pass filter, respectively. The sequence of functions $\left\{u_{n}\right\}(n=0,1,2, \ldots)$ generated from a given function $u_{0}$ by Eq. (6) is called the wavelet packet basis function.

\section{SIMULATION RESULTS}

Figure 4 show the Noisy and denoised signal. Figure 5 illustrates original signal along with the detail plotted for levels one through five. Figure 6 shows the error signal. The convergence rate is illustrated in figure 7

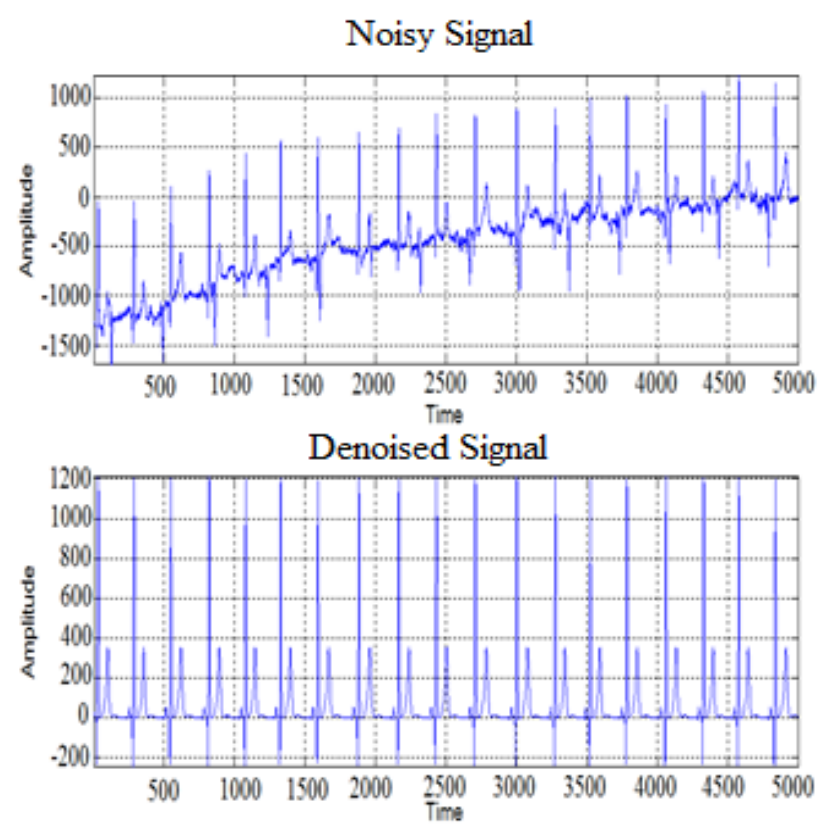

Fig. 4 Noisy and denoised signals
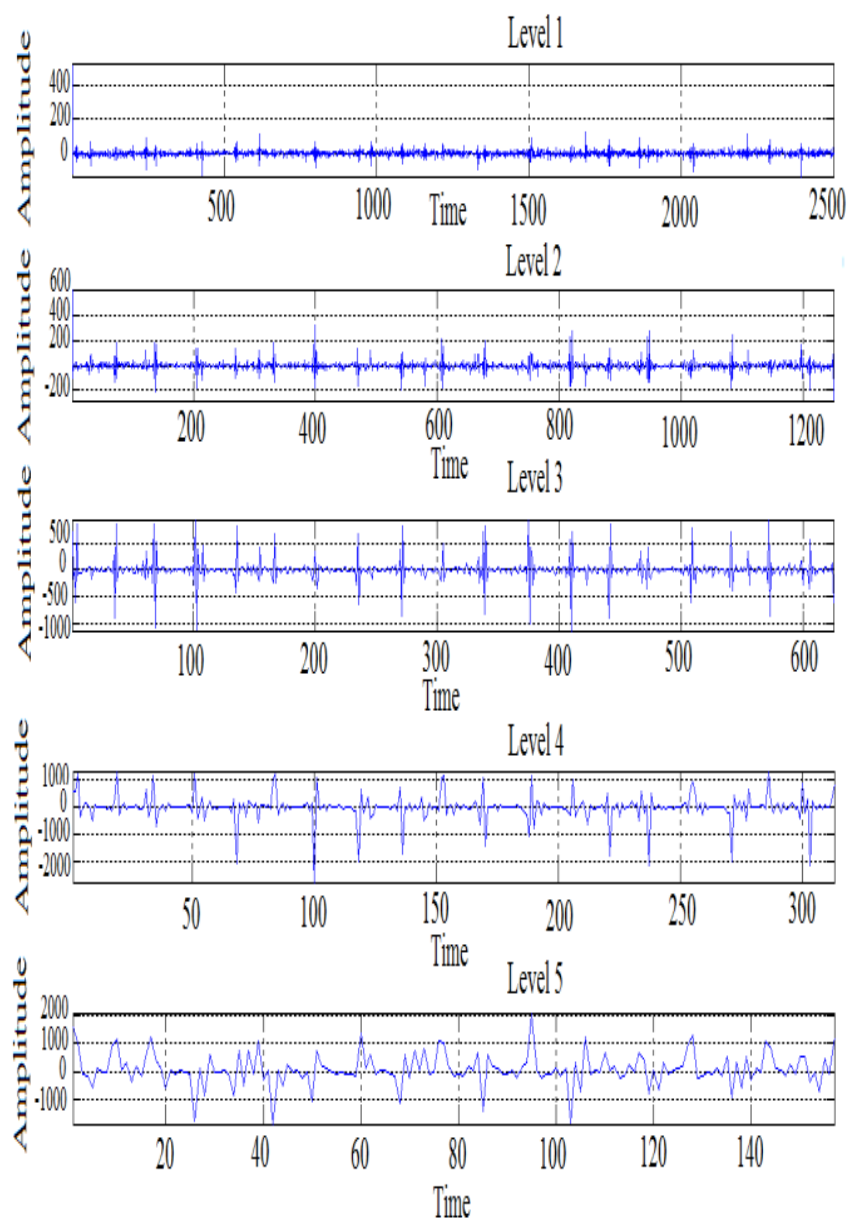

Fig. 5 Original signal along with the detail plotted. 


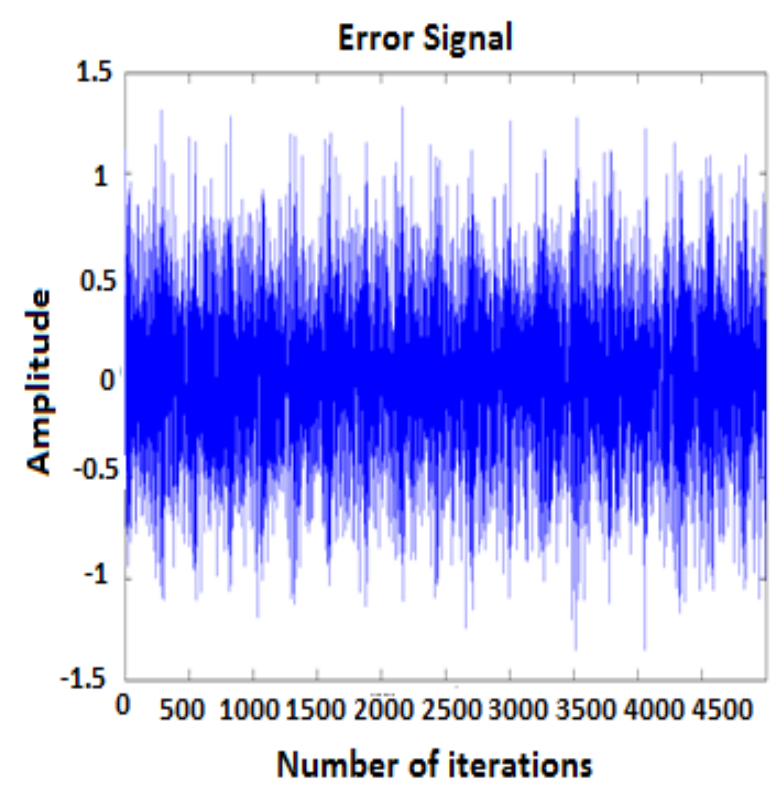

Fig. 6 The effect of increasing number of iterations on error signal

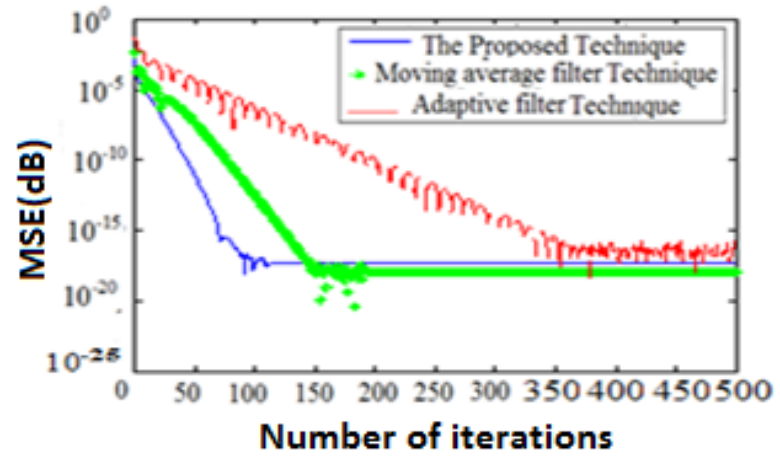

Fig.7 The effect of increasing number of iterations on MSE

Notice that the activity in the details reduces drastically as the scale or the level increases, so we will focus on the details and ignore the rest for now. The analysis in here is to retain the shape changes and getting rid of the noise. To do so, the detail coefficients can be scaled by a "Threshold" as shown in the analysis and the proposed technique sections. Tables 1 and 2 show comparison of different approaches in terms of convergence rate for $\mathrm{N}$ (order of filter) $=10$ and $\mathrm{SNR}=10 \mathrm{~dB}$ respectively

Table 1: Comparison of different approaches at $\mathrm{N}$ (order of filter) $=10$

\begin{tabular}{|c|c|c|c|}
\hline SNR & $\begin{array}{c}\text { Number of iterations } \\
\text { using Adaptive Filter[14] }\end{array}$ & $\begin{array}{c}\text { Number of iterations } \\
\text { using SWMA[2] }\end{array}$ & $\begin{array}{c}\text { Number of iterations using } \\
\text { Proposed Approach }\end{array}$ \\
\hline $5 \mathrm{~dB}$ & $90 \times 10^{4}$ & $130 \times 10^{4}$ & $13 \times 10^{4}$ \\
\hline $10 \mathrm{~dB}$ & $220 \times 10^{4}$ & $240 \times 10^{4}$ & $30 \times 10^{4}$ \\
\hline $15 \mathrm{~dB}$ & $500 \times 10^{4}$ & $300 \times 10^{4}$ & $100 \times 10^{4}$ \\
\hline
\end{tabular}

Table 2: Comparison of different approaches at $\mathrm{SNR}=10 \mathrm{~dB}$

\begin{tabular}{|c|c|c|c|}
\hline $\begin{array}{c}\text { N (order } \\
\text { of filter) }\end{array}$ & $\begin{array}{c}\text { Number of iterations } \\
\text { using Adaptive Filter[14] }\end{array}$ & $\begin{array}{c}\text { Number of iterations } \\
\text { using SWMA[2] }\end{array}$ & $\begin{array}{c}\text { Number of iterations } \\
\text { using Proposed Approach }\end{array}$ \\
\hline 5 & $42 \times 10^{4}$ & $22 \times 10^{4}$ & $13 \times 10^{4}$ \\
\hline 10 & $150 \times 10^{4}$ & $28 \times 10^{4}$ & $20 \times 10^{4}$ \\
\hline 15 & $630 \times 10^{4}$ & $30 \times 10^{4}$ & $28 \times 10^{4}$ \\
\hline
\end{tabular}

Table 1 and 2 showed that the proposed technique achieved faster convergence rate compared with the other techniques.

\section{FPGA REALIZATION}

The proposed design is simulated via FPGA to show how the proposed design achieved excellent results in terms of hardware implementation. The procedures done to clarify the algorithm of the proposed design can be summarized as follow. First, the matlab code was used to determine optimal parameters for wavelet transform. After that, the output of the

Matlab code was converted to fixed point representation. Next step, this fixed point can be used in the code of virtex -II pro.
The importance of these procedures is that the embedded PowerPC has no floating-point unit (FPU), and at the same time FPGAs don't support floating-point either.

Although a FPU could be designed in an FPGA, they are resource intensive, and therefore can feasibly only support sequential operations. Rounding operation was performed on the signals before converting it into binary format. Figure 8 shows the timing diagram for the output signals using proposed design. Table 3 illustrates the resources used for the different designs compared with the proposed one. 


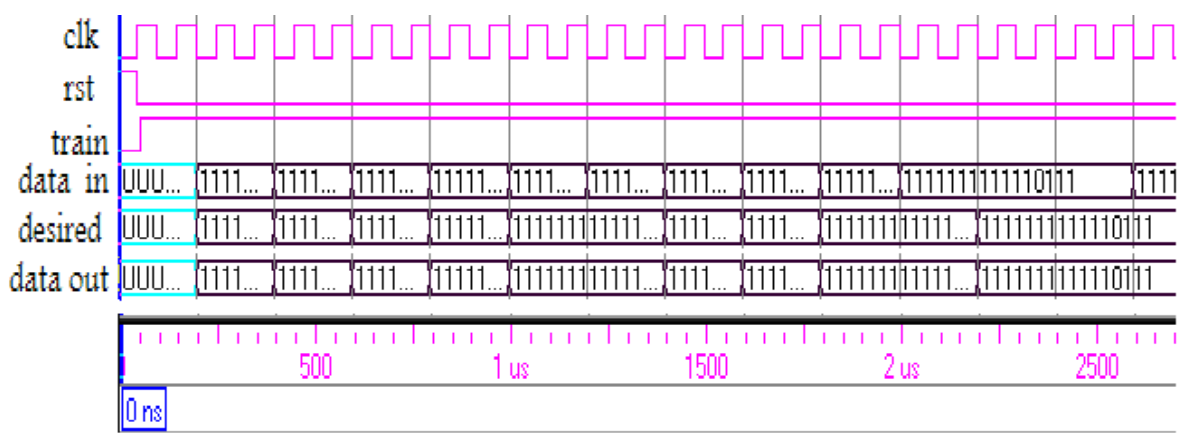

Fig. 8 Timing diagram of ECG signals using the proposed approach

Table 3: Resources used for the different designs compared with the proposed one.

\begin{tabular}{|c|c|c|c|}
\hline Resources & $\begin{array}{c}\text { Utilization for one long } \\
\text { adaptive filter }\end{array}$ & Utilization for SWMA & Utilization for proposed approach \\
\hline IOs & $11.34 \%$ & $11.34 \%$ & $11.34 \%$ \\
\hline Global Buffers & $6.25 \%$ & $5.25 \%$ & $4.15 \%$ \\
\hline Function Generators & $62.77 \%$ & $62.41 \%$ & $60.24 \%$ \\
\hline CLB Slices & $65.75 \%$ & $65.45 \%$ & $63.34 \%$ \\
\hline Dffs or Latches & $8.97 \%$ & $8.93 \%$ & $7.36 \%$ \\
\hline Block Multipliers & $5.33 \%$ & $5.33 \%$ & $5.33 \%$ \\
\hline
\end{tabular}

\section{CONCLUSIONS}

The paper proposed the using of the Discrete Wavelet Transform for denoising an ECG signal with Baseline Wander. Baseline correction has always been a necessary preprocessing in the biomedical signal acquiring and analyzing system. The wavelet transform could remove the noise form the ECG signal efficiently. The difference between the original signal and the denoised one is not of a considerable value which makes this approach to be considered to the biomedical signals that far. In another way, this proposed technique achieved excellent results in terms of convergence rate and mean square error. The proposed design has an efficient realization using FPGA in terms of resources used. All these results give the proposed technique a robust in its domain of search.

\section{REFERENCES}

[1] Momot, A., "Methods of weighted averaging of ECG signals using Bayesian inference and criterion function minimization". Biomedical Signal process. Control,2011 4(2), PP.162-169.

[2] Xiao HU, Zhong XIAO, NI ZHANG, " Removal of baseline wander from ECG signal based on a statistical weighted moving average filter", Journal

of Zeheijang University-SCIENCE C (Computers and electronics),2011, 12(5), PP. 397-403.

[3] M. M. Abo-Zahhad, A. I. Hussien, and A. M. Mohamed, "compression of ECG signal based on compressive sensing and the extraction of significant features," Int. J. communications, Network and system sciences, ,2015, PP. 97-117.

[4] H. Mamaghanian, N. Khaled, D. Atienza and P. Vandergheynst," Compressed sensing for real-time energy-efficient ECG compression on wireless body sensor nodes", IEEE Transactions on Biomedical Engineering, 2011, 58(9), PP. 2456-2466.
[5] H. Fathey,E. Mohamed,A. Mohamed, and W. Anis, "Enchancement of ECG signal", International journal of computer Applications , 2016, 145(7), PP. 12-6.

[6] B. Xhaja, E. Kalluci, and L. Nikolla, "Wavelet transform applied in ecg signal processing", European scientific journal, 2015, 11(12), PP.305-312.

[7] F.chen, Chandrakasan, and Stojanovic, " Design and analysis of a hardware-efficient compressed sensing architecture for data compression in wireless sensors", IEEE journal of solid state circuit , 2012, 47(12), PP. 744-756.

[8] Leski, J.M., Henzel, N., "ECG baseline wander and powerline interference reduction using nonlinear filter bank", 2005 Signal Process., 85(4), PP.781-793.

[9] Hu, X., Xiao, Z., Liu, C.H.,"Reduction Arithmetic for Power Line Interference from ECG Based on Estimating Sinusoidal Parameters". 3rd Int. Conf. on Biomedical Engineering and Informatics, 2010, PP. 2089-2092.

[10] Xu, L.S., Zhang, D., Wang, K.Q., Li, N.M., Wang, X.Y.,"Baseline wander correction in pulse waveforms using wavelet-based cascaded adaptive filter", Computer Biol. Med., 2007, 37(5), PP.716-731.

[11] Chen, H.Y., Huang, M., Jiang, Y.X., Hai, J. "Detection of ST segment in electrocardiogram by wavelet transform". Electr. Mach. Control, 2006 10(5), PP.531533.

[12] Shi, L., Yang, C.Y., Fei, M.R., "Electrocardiogram Rwave and ST segment extraction based on wavelet transform", Chin. J. Sci. Instrum.,2008, 29(4), PP.221227.

[13] S. E Jeroa, Palaniappan Ramua, and S.Ramakrishnanb, "Ecg steganography using curvelet transform", Biomedical signal processing and control, 2015, PP. 519-530. 
[14] Guohua Lu, John-Stuart Brittain, Peter Holland, John Yianni, Alexander L. Green, John F. Stein, Tipu Z. Aziz and Shouyan Wang, "Removing ECG noise from surface EMG signals using adaptive filters", Journal of Neuroscience letters, 2009, 462, PP. 14 - 19.

[15] Sachin Singh and K.L. Yadav, "performance evaluation of different adaptive filters for ECG signal processing", International journal on computer science and engineering, 2006, 02(4), PP. 90- 93.

[16] Wilfried Philips, "Adaptive noise removal from biomedical signals using warped polynomials". IEEE transactions on biomedical engineering, 43(5), May 2013, PP. $480-492$

[17] Abdel-Rahaman AL- Qawasmi, and Khaled Daqrouq, "ECG signal enhancement using wavelet transform". WSEAS transactions on biology and biomedicine, April 2010, 7(2), PP. 62- 72.

[18] J. Mateo, C. Sanchez, C. Vaya, R. Cervigon and J. J. Rieta, "A new adaptive approach to remove baseline wander from ECG recordings using Madeline structure". Computers in Cardiology, 2007, 34, PP. 533 - 536.

[19] Lin and Yue- Der Lin, "An adaptive algorithm for cancelin power line interference in biopotential measurement", Biomedical engineering, basis and communication, December 2004, P.P.350- 354.

[20] Sörnmo, L.,"Time-varying digital filtering of ECG baseline wander", Med. Biol. Eng. Computer, 2013, 31(5), PP. 503-508

[21] Boucheham, B., Ferdi, Y., Batouche, M.C., "Piecewie linear correction of ECG baseline wander: a curve simplification approach", Computer. Methods Programs Biomedical, 2005,78(1), PP.1-10.

[22] Chen, H.Y., Huang, M., Jiang, Y.X., Hai, J., Detection of ST segment in electrocardiogram by wavelet transform", Electr. Mach. Control, 2006, 10(5), PP. 531-533.

\section{AUTHOR PROFILE}

Ashraf Mohamed Ali Hassan was born in 1979 in Giza. He received his B.S.E.E. Degree in electrical Engineering in 2002 with very good with honor from Cairo university. He received his M.Sc. and Ph.D. degree in electrical engineering in 2005 and 2009 respectively, both from Cairo University. He is working as lecturer at October University for modern sciences and arts. He published more than 25 international papers. His research interests include digital signal processing and synthesis of electronic circuits. 